\title{
Changes in distress of psychiatric in-patients after the changeover of junior psychiatric trainees as a function of attachment style: A pilot study
}

\section{David Dodwell ${ }^{\star}$ \\ Toral Thomas** \\ Zeinab lqbal ${ }^{\star \star \star}$}

* Consultant Psychiatrist, Peterborough

\& Fenland Assertive Outreach Teams, Cambridgeshire \& Peterborough Foundation NHS Trust

** ST2 Psychiatrist, Cambridgeshire \& Peterborough Foundation NHS Trust

*** ST5 Psychiatrist, Cambridgeshire \& Peterborough Foundation NHS Trust

UNITED KINGDOM

\begin{abstract}
Background and Objectives: Therapists, including psychiatrists, may act as attachment figures. Rotational training schemes necessitate the regular and frequent ending of therapeutic relationships. The effects on patients are rarely studied. This is a pilot prospective study to evaluate whether relative distress in adult psychiatric in-patients follows change in trainee psychiatrists; whether differences in distress are mediated by patient attachment style; and to provide data for power calculation.

Methods: Twenty adult in-patients were assessed using the Mental Health Inventory 5 (MHI-5) scale before and after changeover of psychiatric trainees; attachment style was assessed prior to the changeover. Qualitative data were also collected.

Results: The average MHI-5 scores improved with time $(\mathrm{p}=0.021)$. Less improvement correlated with higher score on preoccupied attachment (rho $=0.41, \mathrm{p}_{1 \text {-tail }}<0.05$ ). A nonsignificantly stronger improvement was seen with secure/dismissing styles compared to preoccupied/fearful styles $\left(\mathrm{p}_{1 \text {-tail }}=0.08\right)$. Based on these results, a study of at least 87 patients is likely to produce a statistically significant result. Most patients were not aware a staff change was due and would like to be informed by their clinical team.

Conclusions: Relative distress following junior trainee changeover may have a link with patients' attachment styles.
\end{abstract}




\section{Introduction}

Bowlby ${ }^{1-3}$ proposed the concept of attachment and developed attachment theory, based on a cross-fertilisation between psychoanalytic theory and ethology, and informed by direct observations of problems in developmental psychology and psychiatry. He suggested that this model is internalised, linking it to cognitive schemas.

Attachment behaviour results in a person seeking or maintaining proximity to an attachment figure (normally their care-giver) when feeling threatened - including feeling frightened, tired, or ill ${ }^{3}$. It was originally described in the relationship between adult and young; it has since been extended to apply to adult-to-adult relationships, most commonly romantic ones ${ }^{4}$, but including therapist-client ones ${ }^{5}$.

Attachment theory may be relevant to adult mental health in at least 4 ways:

1. Some adult disorders may be conceptualised as primary attachment disorders (e.g. borderline personality disorder) ${ }^{6}$.

2. Some attachment styles may predispose to adult psychiatric disorders, so adult mental health patients may have excess rates of attachment disorder ${ }^{6}$.

3. Much adult mental health provision can only be delivered within the context of some kind of relationship between patient/client and staff/therapist (a therapeutic relationship or working alliance ${ }^{7}$ ). There are clear parallels between attachment and the therapeutic alliance $^{8}$. Psychiatric staff themselves can take on the role of attachment figures in the in-patient population ${ }^{9}$. Insofaras attachment disorders generalise to other relationships, they are likely to affect the efficient delivery of mental health care, causing problems such as dependency, disengagement and malignant alienation ${ }^{9}$. The attachment styles patients develop have been shown to be relevant to their relationship with mental health services ${ }^{10}$. Equally, service organisations which pay no heed to the therapeutic alliance will impede good service delivery: transient junior trainees have higher non-attendance rates at Out-Patient Clinics than more permanent staff ${ }^{11}$.

4. Attachment style may influence helpseeking and symptom reporting ${ }^{12}$.

At the time of this study, the practice was that the majority of junior doctors working in psychiatry were trainees who changed post every 6 months. The junior trainee was usually the day-to-day point of medical contact for patients on the ward and this en masse changeover provided an ideal opportunity to study its effects on patients. At the time of the study, British junior trainees in psychiatry were called Senior House Officers (SHOs).

\section{Aims of this study}

Our aim was to conduct a pilot study looking at the attachment styles of adult inpatients in the two local general psychiatric wards, to see if there was any differential change in their mental health during the junior trainee changeover period. We hypothesized that, overall, patients would get better with the passage of time, but that those with secure or dismissing attachment styles would be less affected by staff change than those with preoccupied or fearful attachment styles, who might be more susceptible to delay or interruption in overall improvement when staff changed. As any effect size, if present, was not known, it was not possible to do a statistical power calculation ${ }^{13}$, but this pilot study would allow a power calculation for future work. 


\section{Methods}

\section{Design}

The study is a prospective A B study without a control or intervention group. Measures took place during two periods - one being in the week prior to junior trainee changeover and the other after 1 week but within two weeks of changeover, to allow the participants time to meet the new trainees.

\section{Participants}

All adult in-patients in the two local general adult psychiatry wards were potentially included in the study. The wards were mixed-sex open wards for psychiatric patients aged between 17 and 65 years of age and provided hospital back-up for the adult Community Mental Health Teams, the Rehabilitation and Assertive Outreach Teams and the Community Alcohol Team.

\section{Exclusion criteria}

Patients with an insufficient command of the English language, patients unable or unwilling to consent, and those under the age of 16 years were excluded. As preparation for the study all the consultant psychiatrists were contacted to see if there were particular patients who might be too unwell to participate. In order to raise awareness of the project two researchers went to the morning ward community meeting to discuss the project and put up posters with some information. The ward staff were also given printed information about the study.

Patients were interviewed by one of two researchers to prevent a researcher from interviewing a patient with whom they already had regular clinical involvement. One interview was performed in the week prior to the trainee changeover and the second interview was done between one and 2 weeks after the changeover, to allow the patients time to meet the new trainees. The rating scales were either read out to the patients or completed by the patients themselves, depending on patient preference.

\section{Measures}

Given the mixed diagnostic group and the fact that we were interested in overall state, we used the Mental Health Inventory 5 item (MHI-5) questionnaire ${ }^{14}$ as a measure of non-specific distress ${ }^{15,16}$. The MHI-5 scale is a subset of the SF-36 Health Survey instrument ${ }^{17}$ and the 38 item Mental Health Inventory ${ }^{18}$ : it consists of 5 items with responses on a 6-point scale ranging from "all of the time" to "none of the time." The possible score range is 5 to 30 with higher scores indicating better mental health. The MHI- 5 has well-established reliability and validity in general population studies; whilst not primarily a diagnostic instrument, as a screening test it agrees well with a diagnostic interview $^{14}$; it has good internal consistency (Cronbach's alpha 0.84) ${ }^{19}$. It is also short and easy to understand. The MHI-5 was administered before and after the staff changeover.

The measurement of attachment often relies on lengthy interviews or questionnaires - these would not be suitable for acute in-patients who may have limited concentration. We therefore used a technique described by Bartholomew \& Horowitz ${ }^{20}$ as self-report attachment style prototypes: patients were presented with four vignettes (corresponding to secure, dismissing, preoccupied, or fearful styles of attachment) and were asked to rate their agreement with each on a 7 point scale (low score $=$ high agreement) to 
produce a score for each vignette (vignette score); they were then asked to pick one of the four styles as being most representative of themselves (forced choice rating). This model of attachment postulates that secure and dismissing styles share a positive view of self with low dependence on others, and therefore in this study were expected to be more resilient to distress at breaking attachment bonds. The attachment rating was carried out at the first interview.

At the second interview, following changeover, the patients were asked the following five questions:

1. Were you aware that the SHOs were going to change over?

2. What is the best way to inform patients about the changeover?

3. Were you upset in any way due to the changeover?

4. Is there anything that would make changeovers less upsetting for the patients?

5. Have you met your new SHO yet?

Data were mainly analysed using SPSS $®$ for Windows ${ }^{\circledR}$. Some data were analysed by hand $^{21}$. For all tests we used $\mathrm{p}=0.05$ as our level of statistical significance.

Scores on MHI-5 at the first interview were subtracted from those at the second interview to give a change score. For some analyses, the participants were divided into two main groups - positive view of self (comprising forced-choice secure and dismissive styles) and negative view of self (comprising forced-choice preoccupied and fearful attachment styles).

Three of the five qualitative interview questions produced yes/no answers and two of the authors looked at the responses to the other two questions to find common themes.

\section{Results}

\section{Descriptive}

Four patients were excluded from the study - three because English was not their first language and one was deemed to be too thought disordered to participate. Twentythree patients initially consented to the study; 20 patients completed both interviews, while three patients refused to have a second interview and were not included in the final results.

The median age was 37 years (range $18-$ $61)$. Eight were female and 12 were male. Fifteen had been in hospital for 3 months or less. The single commonest diagnosis was schizophrenia (7), followed by other non-affective psychoses (4), bipolar affective disorder (3), depressive illnesses (2), unclear (2), alcohol dependence (1), and schizophrenia plus drug abuse (1).

\section{Distress}

Pre-changeover, MHI-5 scores had a median of 20 (range 5-29) whilst after changeover the MHI-5 scores showed a median of 21.5 (range 11-29). The change score median was +2 , range +11 to -6 . Comparing the MHI-5 score pre and post changeover using the Wilcoxon signed-ranks test gave $\mathrm{z}$ $=2.04, \mathrm{p}=0.021$, showing that the patients' distress reduced over time.

\section{Attachment}

On forced-choice, 5 patients selected secure, 3 each dismissing and preoccupied, and 9 chose fearful attachment styles. The numerical scores on each vignette were compared with the forced-choice categories in two ways. The mean ranked scores on the 
vignette were compared across the four forced-choice groups (once each for each vignette) using the Kruskal-Wallis one-way analysis of variance (Siegel, 1956) with the hypothesis that there would be a difference. The result for scores on the 'secure' vignette was $\mathrm{H}=7.40$ (corrected for ties), $\mathrm{p}_{1 \text {-tail }}<0.05$; for the 'dismissing' vignette scores, $\mathrm{H}=9.30$, $\mathrm{p}_{1 \text {-tail }}<0.05$, for 'preoccupied' scores $\mathrm{H}=4.65$, $\mathrm{p}_{1 \text {-tail }}<0.10$; and for 'fearful' scores, $\mathrm{H}=10.04$, $\mathrm{p}_{1 \text {-tail }}<0.01$. These results indicate good agreement between the forced choice and vignette score ratings. Secondly, a series of Mann-Whitney U tests were performed comparing the forced-choice with the vignette score results, with the hypothesis that there would be a positive association between the two. Comparing the forced-choice 'secure' versus the other 3 choices against the 'secure' vignette score, $\mathrm{z}=2.44, \mathrm{p}_{1 \text {-tail }}<0.01$; for 'dismissing' versus other choices on 'dismissing' score $\mathrm{z}=2.01, \mathrm{p}_{1 \text {-tail }}<0.05$; for 'preoccupied' versus other choices on 'preoccupied' score $z=1.67$ (corrected for ties), $p_{1 \text {-tail }}$ $<0.05$; and for 'fearful' versus other choices on 'fearful' score $\mathrm{z}=2.74 \mathrm{p}_{1 \text {-tail }}<0.005$.

The vignette scores were also correlated with each other (table 1). These show that there are generally weak intercorrelations between the scores, with the exception of strong agreement between secure and dismissing and strong disagreement between secure and fearful vignette scores. The former supports the hypothesis that secure and dismissing styles share a common core of high self-worth.

\section{Relationship between distress change and attachment style}

Table 1 also lists the correlations between the four vignette scores and the MHI-5 change scores. The correlations were all in the expected directions, although only one reached statistical significance - that between MHI-5 change and the preoccupied vignette score.

The forced-choice attachment styles were pooled into two groups - secure \& dismissing (i.e. positive view of self) versus preoccupied \& fearful (negative view of self) and compared with the MHI-5 change score using the Mann-Whitney test, resulting in $\mathrm{U}=62, \mathrm{z}=-1.04 \mathrm{p}_{1 \text {-tail }}=0.08$. The numbers involved were extremely small. Using the same proportion of people doing better in the secure $\&$ dismissing group (0.68), a power calculation indicates that identical results would have been statistically signifi-

Table 1

Correlations of vignette scores with each other and with MHI-5 change score

\begin{tabular}{lcccc} 
& Secure & Dismissing & Preoccupied & Fearful \\
\hline Dismissing & $0.60 * *$ & & & \\
\hline Preoccupied & 0.06 & -0.15 & -0.06 & 0.35 \\
\hline Fearful & $-0.69 * *$ & -0.39 & $0.41 \#$ & -0.20 \\
\hline MHI-5 change & -0.29 & & & \\
\hline
\end{tabular}

Figures are Spearman's rho.

nb low score on vignette indicates agreement, high MHI-5 change indicates improvement.

$* * \mathrm{p}_{2 \text {-tail }}<0.01$.

$\# \mathrm{p}_{1-\text { tail }}<0.05$. 
cant at $\mathrm{p}=0.05$ if the number of patients had been at least 87 (for power $/ \beta=0.8$ ), or 117 (for power/ $\beta=0.9$ ).

\section{Qualitative results}

Eighteen of the twenty patients in the study completed the five questions. Only three $(17 \%)$ were aware of the junior trainee (SHO) changeover and none admitted to finding it upsetting. By one week after changeover only nine $(45 \%)$ said they had met their new SHO.

When asked about how best to be informed of the junior trainee changeover, most patients $(\mathrm{n}=11)$ advocated a direct approach either verbally or in writing. One suggestion was to write about the forthcoming changeover on a board in the ward. Nearly half $(n=8)$ of patients wanted to be informed in person about the changeover by a doctor or nurse.

\section{Discussion}

Making reliable, valid measurements in this patient group is quite challenging. The results show that it is possible to measure change in distress over a relatively short period of time and to make some tentative links between such change and attachment style. It also shows that patients were largely unaware of routine staff changeovers and have simple, practical views about being informed.

Apart from the exclusion criteria, the patients were unselected general adult mental health in-patients. Most of those approached agreed to before and after interviews. They are likely to be representative of British inpatients at the time of the study, although given the long-term trends in bed reduction and changes in service provision, this could change in the future. Increasingly short durations of in-patient care reduce the opportunities for attachment and therefore for distress at the ending of relationships with staff.

The measure of distress chosen was designed for predominantly 'neurotic' problems in out-patient and community settings ${ }^{14}$, not for those sufficiently ill to require hospitalisation, many of whom had a diagnosis of psychotic illness. However, it is based on the concept of non-specific distress ${ }^{15}$, which is applicable to this population and forms a 'lowest common denominator' measure of mental disorder. We wanted a brief measure which was not diagnosis-specific. We tried to make it more accessible by giving patients a choice of reading the form and self-rating or having it read to them. Our results show that there was no ceiling effect and only one 'floor' score, suggesting it has covered the relevant area of distress. The fact that there was a significant improvement after only 2 weeks also shows sensitivity to change.

Similar points apply to the measurement of attachment style. Many of the techniques used have been quasi-experimental (e.g. the 'strange situation' ${ }^{22}$ ), or involved complex procedures and/or questionnaires aimed at cooperative, normal populations or 'captive' groups such as psychology students ${ }^{4,20}$. Trying to capture the interest and limited concentration of in-patients requires a briefer technique, as well as one applicable to all relationships rather than those specific to adult-young or to romantic settings. For this reason, we chose the vignette procedure of Bartholomew \& Horowitz ${ }^{20}$. Patients had a choice of reading material themselves or having it read to them. Our results showed that there was internal agreement between the forced-choice and vignette score forms of the assessment, supporting the reliability of the technique. The fact that 1 of the 4 vi- 
gnette scores correlated with MHI-5 change indicates some predictive validity; the other 3 vignette scores all showed a trend in the hypothesised direction, and the pooled forced-choice groups (secure \& dismissing versus preoccupied \& fearful) also showed a trend in the hypothesised direction. The numbers involved were small, but do provide data for power calculations for a substantive future study.

There are a number of factors which are likely to dilute potential positive findings. The assumption was that the junior trainee could be a significant attachment figure for an in-patient. Two key elements in that assumption are the importance/role of the junior trainee and the amount of contact. The original conception was based on historic psychiatric practice, where a significant part of the junior trainee's role was working on one ward with in-patients. In this study, many juniors had significant duties elsewhere and themselves lacked an attachment to the wards and to the in-patients of their team. This meant that their ability to spend time with patients was limited. It is notable that less than half the patients said they had met "their' new SHO after 1 week, although this could be partly due to patients having met the junior trainee but not knowing that that was their role/title. The ability to form relationships also depends on the duration of admission. Seventy-five percent of the patients had been in hospital 3 months or less, allowing a relatively brief time for relationship-building, particularly if contact over that time was infrequent and brief.

The attachment concept originally applied to an adult 'primary care-giver' and a juvenile recipient of care. In this study we are assuming that the patient occupies the recipient role, but there are numerous contenders for the role of care-givers in this context. Some patients may have seen as much of their consultant as of the trainee while in hospital and may have had a much longer relationship with their consultant, particularly in teams working with chronic psychosis (Rehabilitation and Assertive Outreach); absence of a consultant has been proposed as a destabilising factor ${ }^{23}$. For inpatient care, nursing staff are the most numerous and most visible group of staff: nurses have been described as the most helpful staff group by in-patients ${ }^{24}$, whilst psychiatrists were rated as the least helpful. Other staff are also vital in ward settings, and of course patients are encouraged to maintain contact with family and friends, so their usual attachment figures should continue to be available. For these reasons, the attachment-figure role of a junior trainee is likely to be limited, but they were studied as the end of their relationships with patients is predictable and recurrent. It may be that attachment is more relevant to long-term community-based work, where the most frequent contact is usually with non-medical staff. It would be interesting to do similar studies relating to change in consultant psychiatrists or in primary (key) nurses.

Our research was done on a small number of patients. Future research will hopefully involve a much larger sample population and allow for correlations to be looked for between change scores on MHI-5 and patients' length of time in current care setting, as this is likely to be an important confounding variable.

As psychiatrists we are always looking for ways to improve our therapeutic relationships with patients. Trainee psychiatrists form an integral part of many teams, but the movement between teams necessary to expose trainees to a variety of clinical experience makes them a transient part of any team. This changeover is sometimes not even discussed with patients and may lead to slower 
reduction in distress. Patients need to have clearer explanation and communication. Although not all therapeutic relationships involve attachment ${ }^{25}$, it is clear that attachment issues can be clinically important ${ }^{25,26}$. More research could usefully be done on identifying if certain patients are particularly vulnerable to staff change; if certain staff roles/relationships are particularly significant; and how attention to attachment issues can improve caregiving ${ }^{26}$.

\section{Acknowledgments}

The authors are extremely grateful to Dr Linda Sharples of the MRC Biostatistics Unit and R\&D Unit, Papworth NHS Foundation Trust, Cambridge, UK, for statistical advice, and to the patients and ward staff involved.

\section{Contributors}

D. Dodwell designed the study, wrote the protocol, obtained ethical permission, and edited the typescript. T. Thomas and $\mathrm{Z}$. Iqbal drafted the typescript; T. Thomas did most of the statistical analysis. All authors have approved the final typescript.

\section{References}

1. Bowlby J. The making and breaking of affectional bonds. I. Aetiology and psychopathology in the light of attachment theory. Brit J Psychiatry 1997; 130: 201-210.

2. Bowlby J. The making and breaking of affectional bonds. II. Some principles of psychotherapy. Brit J Psychiatry $1997 ; 130: 421-431$.

3. Bowlby J. Attachment and Loss. Volume I: Attachment. London: Penguin; 1991.
4. Hazan C, Shaver P. Romantic love conceptualized as an attachment process. J Personality Soc Psychol 1987; 52: 511-524.

5. Fonagy P, Leight T, Steele M, Steele H, Kennedy R, Mattoon G, et al. The relation of attachment status, psychiatric classification, and response to psychotherapy. J Consult Clin Psychology 1996; 64: 22-31.

6. Holmes J. John Bowlby \& Attachment Theory. London: Routledge; 1993.

7. Horvath AO, Bedi RP. The Alliance. In: Norcross JC (ed). Psychotherapy Relationships That Work. New York: Oxford University Press; 2002.

8. Mackie AJ. Attachment theory: its relevance to the therapeutic alliance. Brit J Med Psychol 1981; 54: 203-212.

9. Adshead G. Psychiatric staff as attachment figures. Understanding management problems in psychiatric services in the light of attachment theory. Brit J Psychiatry 1998; 172: 64-69.

10. Goodwin I, Holmes G, Cochrane R, Mason O. The ability of adult mental health services to meet clients' attachment needs: The development and implementation of the Service Attachment Questionnaire. Psychol Psychother Theor Res Pract 2003; 76: 145-161.

11. McIvor R, Ek E, Carson J. Non-attendance rates among patients attending different grades of psychiatrist and a clinical psychologist within a community mental health clinic. Psychiatric Bull 2004; 28: 5-7.

12. Ciechanowski PS, Walker EA, Katon WJ, Russo JE. Attachment theory: a model for health care utilization and somatisation. Psychosom Med 2002; 64: 660-667.

13. Cohen J. Quantitative methods in psychology: a power primer. Psychol Bull 1992; 112: 155-159.

14. Berwick DM, Murphy JM, Goldman PA, Ware JE, Barsky AJ, Weinstein MC. Performance of a five-item mental health screening test. Med Care 1991; 29: 169-176.

15. Dohrenwend BP, Shrout PE, Egri G, Mendelsohn FS. Nonspecific psychological distress and other dimensions of psychopathology. Arch Gen Psychiatry 1980; 37 : 1229-1236.

16. Briggs L, Macleod AD. Demoralisation - a useful conceptualization of non-specific psychological distress among refugees attending mental health services. International J Soc Psychiatry 2006; 52: 512-524.

17. Ware JE, Gandek B. Overview of the SF-36 health survey and International Quality of Life Assessment (IQOLA) project. J Clin Epidemiol 1998; 51: 903-912.

18. Veit CT, Ware JE. The structure of psychological distress and well-being in general populations. J Consult Clin Psychol 1983; 51: 730-742. 
19. McCabe CJ, Thomas KJ, Brazier JE, Coleman P. Measuring the mental health status of a population: a comparison of the GHQ-12 and the SF-36 (MHI-5). Brit J Psychiatry 1996; 169: 516-521.

20. Bartholomew K, Horowitz LM. Attachment styles among young adults: a test of a four category model. J Personality Soc Psychology 1991; 61: 226-244.

21. Siegel S. Nonparametric Statistics for the Behavioral Sciences. Tokyo: McGraw-Hill Kogakusha Ltd.; 1956.

22. Ainsworth M, Blehar M, Waters E, Wall S. Patterns of Attachment: assessed in the strange situation and at home. Hillsdale NJ: Erlbaum; 1978.

23. Persaud RD, Meux CJ. The psychopathology of authority and its loss: the effect on a ward of losing a consultant psychiatrist. Br J Med Psychol 1994; 67: 1-11.

24. Rogers A, Pilgrim D, Lacey R. Experiencing Psychiatry - users' views of services. Houndmills, Basing- stoke: Macmillan Press in association with MIND publications; 1993.

25. Ma K. Attachment theory in adult psychiatry. Part 2: Importance to the therapeutic relationship. Adv Psychiatric Treatment 2007; 13: 10-16.

26. Berry K, Drake R. Attachment theory in psychiatric rehabilitation: informing clinical practice. Adv Psychiatric Treatment 2010; 16: 308-315.

\author{
Author for correspondence: \\ David Dodwell \\ Lucille van Geest Centre, Thorpe Road \\ Peterborough PE3 6DA, UK \\ Telephone: 01733318124 \\ Fax: 01733318140 \\ E-mail: david.dodwell@cpft.nhs.uk
}

